\title{
Soroprevalência de Borrelia burgdorferi latu sensu associada à presença de carrapatos em cães de áreas rurais do Estado do Rio de Janeiro, Brasil
}

\author{
Seroprevalence of Borrelia burgdorferi latu sensu associated with dog ticks in \\ rural areas of the Rio de Janeiro State, Brazil \\ Lucia Helena O'Dwyer ${ }^{1}$ Cleber Oliveira Soares ${ }^{2}$ Carlos Luiz Massard ${ }^{3}$ \\ José Carlos Pereira de Souza ${ }^{4}$ Walter Flausino ${ }^{5}$ Adivaldo Henrique da Fonseca ${ }^{6}$
}

\section{RESUMO}

Pesquisou-se a presença de anticorpos contra Borrelia burgdorferi latu sensu em cães de áreas rurais de sete municípios do Estado do Rio de Janeiro, pelo ensaio imunoenzimático indireto, associando-se os resultados com a presença de carrapatos nestes animais. De 199 cães examinados, $15,58 \%$ estavam positivos, com títulos que variaram de $400(13,57 \%)$ a 1600 (0,5\%). Os casos positivos se distribuíram uniformemente nos sete municípios. Não houve diferença estatística $(P>0,05)$ de soropositivos entre as faixas etárias dos cães acima de seis meses. Carrapatos foram encontrados e coletados em 71 (35,68\%) cães, dos quais $24,1 \%$ estavam infestados com Amblyomma cajennense, $13,6 \%$ com Rhipicephalus sanguineus, $2,5 \%$ com Amblyomma aureolatum e 1,5\% com Amblyomma ovale. Dos animais soropositivos para B. burgdorferi, $38,7 \%$ apresentavam A. cajennense e 22,6\% apresentavam $\boldsymbol{R}$. sanguineus, não havendo, entretanto, correlação positiva entre a presença do carrapato e sorologia positiva.

Palavras-chave: Borrelia burgdorferi, Amblyomma sp., Rhipicephalus sanguineus, carrapatos, cães.

\section{ABSTRACT}

The prevalence of antibodies against Borrelia burgdorferi latu sensu was investigated in dogs from rural areas of seven towns of the Rio de Janeiro State, Brazil, using an indirect enzyme-linked immunosorbent assay. All dogs were also examined for the presence of ticks. From the 199 dogs, $15.58 \%$ were seropositive, with titers that ranged from $400(13.57 \%)$ to $1600(0.5 \%)$. The positive cases were equally distributed among the seven studied towns. No differences ( $P$ $>0.05)$ were found among the age groups of over six months old. Ticks were found and collected from 71 (35.68\%) dogs. From this total, $24.12 \%$ were infested with Amblyomma cajennense, $13.6 \%$ with Rhipicephalus sanguineus, $2.5 \%$ with Amblyomma aureolatum and $1.5 \%$ with Amblyomma ovale. Amblyomma cajennense was found in $38.7 \%$ and $\boldsymbol{R}$. sanguineus in $22.6 \%$ of the dogs with $\boldsymbol{B}$. burgdorferi antibodies, although no positive correlation between the ticks parasitism and positive serology was found.

Key words: Borrelia burgdorferi, dogs, ticks, Amblyomma sp., Rhipicephalus sanguineus.

\section{INTRODUÇÃO}

A borreliose de Lyme, doença causada pela espiroqueta Borrelia burgdorferi é transmitida por carrapatos e pode acometer seres humanos e animais (HOSKINS, 1991). Os principais vetores desta bactéria são os carrapatos Ixodes scapularis nos Estados

\footnotetext{
${ }^{1}$ Médico Veterinário, Professor Assistente Doutor, Departamento de Parasitologia, Instituto de Biociências, Universidade Estadual Paulista, 18618-000, Botucatu, SP. E-mail: odwyer@ibb.unesp.br. Autor para correspondência.

${ }^{2}$ Médico Veterinário, Pesquisador, Embrapa Gado de Corte, CP154, 79002-970, Campo Grande, MS.

${ }^{3}$ Médico Veterinário, Professor Titular, Departamento de Parasitologia Animal, Instituto de Veterinária da Universidade Federal Rural do Rio de Janeiro (UFRRJ).

${ }^{4}$ Médico Veterinário, Delegacia Federal de Agricultura do Estado do Rio de Janeiro, Ministério da Agricultura, Pecuária e Abastecimento.

${ }^{5}$ Biólogo, Departamento de Parasitologia Animal, Instituto de Veterinária da UFRRJ.

${ }^{6}$ Médico Veterinário, Professor Titular, Bolsista do CNPq, Departamento de Epidemiologia e Saúde Pública, UFRRJ, Km 47, 23890-970, Seropédica, RJ. E-mail: adivaldo@ufrrj.br.
} 
Unidos da América (EUA) e Ixodes ricinus na Europa (McKENNA et al., 1995). Contudo, outras espécies podem transmitir o agente, dependendo da região fisiográfica (SOARES et al., 2000).

Estudos soroepidemiológicos têm sido conduzidos no mundo inteiro para determinação da prevalência de anticorpos contra $\boldsymbol{B}$. burgdorferi latu sensu em cães e da importância destes animais na epidemiologia dessa doença (McKENNA et al., 1995; WRIGHT et al., 1997; STEFANCIKOV et al., 1998). O conhecimento da soroprevalência em cães pode constituir-se em um importante indicador da dispersão do agente etiológico (HOSKINS, 1991), bem como indicar o risco de exposição humana (LINDENMAYER et al., 1991). Atualmente, cães são considerados animais sentinelas para a borreliose de Lyme, cuja prevalência correlaciona-se positivamente com aquela verificada para seres humanos, na mesma área (MERINO et al., 2000; OLSON et al., 2000).

Em cães, a infecção é usualmente assintomática. Entretanto, podem ser observados sinais envolvendo o sistema músculo-esquelético, com quadro de artrite progressiva (LEVY \& DREESEN, 1992; STRAUBINGER et al., 1998; STRAUBINGER, 2000).

O diagnóstico da borreliose de Lyme é feito principalmente por meio de testes sorológicos, associado a sinais clínicos e a fatores epidemiológicos, já que seu encontro em líquidos orgânicos e secreções é difícil (STEERE, 1989). Com a utilização da técnica de reação em cadeia da polimerase, foi possível detectar DNA de B. burgdorferi em urina de cães (BAUERFEIND et al., 1998), em amostras de membranas sinoviais, pele, fluido cérebro-espinhal, bexiga, coração e medula óssea de cães com infecção sintomática (HOVIUS et al., 1999b; STRAUBINGER, 2000).

No Brasil, a borreliose de Lyme já foi diagnosticada em animais (FONSECA et al., 1996) e em humanos (YOSHINARI et al., 1997). JOPPERT et al. (2001) encontraram, em cães da cidade de São Paulo, $9,7 \%$ de sororeagentes pelo ensaio de imunoadsorção enzimática (ELISA) indireto; sendo que destes, 69,7\% possuíam histórico de contato prévio com carrapatos. Em cães de área urbana, procedentes de municípios da Baixada Fluminense, Estado do Rio de Janeiro, SOARES et al. (1999a) reportaram 20\% de animais soropositivos utilizando o teste ELISA indireto.

Várias espécies de carrapatos podem ser encontradas parasitando cães no Brasil de acordo com a região estudada. MASSARD et al. (1981) e O`DWYER et al. (2001) constataram que cães criados em áreas urbanas eram parasitados principalmente por Rhipicephalus sanguineus, enquanto cães de áreas rurais apresentavam, preponderantemente, espécies de Amblyomma (A. ovale, A. tigrinum, A. aureolatum e A. cajennense).

Os carrapatos do gênero Amblyomma têm potencial de transmitir diversos agentes patogênicos, para cães e seres humanos. Sua possível participação na transmissão de B. burgdorferi no Brasil necessita de estudos. Além disto, B. burgdorferi e espécies correlatas já foram isoladas de carrapatos do gênero Amblyomma (BARBOUR et al., 1996; SOARES et al., 2000).

O presente estudo teve como objetivos avaliar a ocorrência de anticorpos anti-B. burgdorferi e verificar quais espécies de carrapatos ocorrem em cães de áreas rurais do Estado do Rio de Janeiro, correlacionando a detecção de anticorpos contra a espiroqueta com a presença do carrapato.

\section{MATERIAL E MÉTODOS}

\section{Amostras de soro}

Foi efetuada a colheita de sangue de 199 cães em sete municípios do Estado do Rio de Janeiro: Seropédica, Itaguaí, Paracambí, Mangaratiba, Barra do Piraí, Piraí e Miguel Pereira. Foram escolhidas áreas afastadas dos centros urbanos, com características rurais e com presença de matas secundárias. A amostragem foi realizada por conveniência e facilidade de acesso às propriedades.

As amostras de soro obtidas do sangue dos animais foram submetidas à pesquisa de anticorpos da classe IgG contra B. burgdorferi latu sensu por meio do teste ELISA indireto segundo SOARES et al. (1999b).

Todos os cães foram examinados, especialmente nas orelhas e coxins plantares e palmares, para detecção da presença de carrapatos. Os exemplares imaturos, ingurgitados, foram coletados e mantidos em estufa incubadora para BOD a $27^{\circ} \mathrm{C}$ e $80 \%$ de umidade relativa, para que pudessem completar o seu desenvolvimento para posterior identificação. Os carrapatos adultos colhidos diretamente no animal ou obtidos a partir de forma imatura foram conservados em álcool a 70\% e identificados segundo a chave de ARAGÃO \& FONSECA (1961).

O estudo das associações entre as variáveis de interesse foi realizado por meio do teste de Goodman (GOODMAN, 1964; 1965) e do teste exato de Fisher (FISHER, 1993), com nível de significância de 5\%.

\section{RESULTADOS}

Neste estudo, 63 propriedades foram visitadas e 199 cães examinados em sete municípios 
do Estado do Rio de Janeiro, sendo 15 cães em Seropédica, 71 em Itaguaí, 28 em Paracambí, 22 em Mangaratiba, 25 em Barra do Piraí, 28 em Piraí e 10 em Miguel Pereira. As coletas foram realizadas de janeiro de 1997 a junho de 1998 e o número de animais examinado variou de um a 12 cães por propriedade, com média de aproximadamente quatro animais. A grande maioria dos animais adentrava as matas para caçar. Os animais foram separados em quatro faixas etárias, sendo: cães com menos de seis meses de idade, cães com idade variando de seis meses a dois anos, cães com idade de dois a cinco anos e cães com idade superior a cinco anos.

Dos 199 animais estudados, $15,58 \%$ foram positivos com títulos de $400(13,57 \%) ; 800(1,51 \%)$ e $1.600(0,5 \%)$. A distribuição dos casos positivos, segundo a região, encontra-se na tabela 1. Apesar das regiões de Miguel Pereira, Piraí e Itaguaí terem apresentado maiores prevalências, $30,0 \%, 21,4 \%$ e $21,1 \%$ respectivamente (Tabela 1 ), não houve diferença estatística $(\mathrm{P}>0,05)$ entre os municípios pesquisados. Em relação à faixa etária, nenhum cão com menos de seis meses apresentou anticorpos contra- B. burgdorferi e a partir desta faixa etária

Tabela 1 - Anticorpos anti-Borrelia burgdorferi em cães de áreas rurais do Estado do Rio de Janeiro.

\begin{tabular}{lcc}
\hline \multirow{2}{*}{ Município } & \multicolumn{2}{c}{ Número de cães } \\
\cline { 2 - 3 } & Examinados & Soropositivos (\%) \\
\hline Seropédica & 15 & 13,31 \\
Itaguaí & 71 & 21,1 \\
Paracambí & 28 & 7,1 \\
Mangaratiba & 22 & 4,5 \\
Barra do Piraí & 25 & 8,0 \\
Piraí & 28 & 21,4 \\
Miguel Pereira & 10 & 30,0 \\
\hline
\end{tabular}

todas as demais (de 6 meses a mais de cinco anos) foram igualmente acometidas, sem diferença estatística entre elas $(\mathrm{P}>0,05)$. Cães com idade superior a cinco anos foram os que tiveram maior percentual de positividade $(26,5 \%)$.

A distribuição de carrapatos, em cada município, pode ser observada na tabela 2. Carrapatos foram retirados em $71(35,68 \%)$ dos cães examinados, dos quais $24,1 \%$ estavam infestados por $\boldsymbol{A}$. cajennense, 13,6\% por $\boldsymbol{R}$. sanguineus, 2,5\% por $\boldsymbol{A}$. aureolatum e $1,5 \%$ por $\boldsymbol{A}$. ovale. Alguns animais apresentaram mais de uma espécie de carrapato. Em relação aos estádios recuperados, no caso de $\boldsymbol{R}$. sanguineus, A. aureolatum e A. ovale, somente adultos foram encontrados sobre os animais. Já em relação a A. cajennense, foram recuperados sobre os animais preponderantemente ninfas e larvas em grande quantidade, fixadas principalmente nas orelhas dos animais.

Quando relacionamos a presença de carrapatos com a presença de anticorpos contra $\boldsymbol{B}$. burgdorferi observamos que $A$. cajennense foi detectado em $38,7 \%$ dos cães sorologicamente positivos (Tabela 3) e R. sanguineus em 22,6\% (Tabela 3) . No entanto, não houve associação entre a presença de anticorpos contra $\boldsymbol{B}$. burgdorferi e o parasitismo por carrapatos.

\section{DISCUSSÃO}

A prevalência de anticorpos anti-B. burgdorferi encontrada em cães varia de acordo com a região fisiográfica (MAGNARELLI et al., 1985; AZUMA et al., 1994; WRIGHT et al., 1997; SALINASMELENDEZ et al., 1999; SOARES, et al., 1999a; 2000; JOPPERT et al., 2001) sendo mais elevada em áreas endêmicas para a borreliose humana (LINDENMAYER et al., 1991; MERINO et al., 2000; OLSON et al., 2000).

Tabela 2 - Número e percentual de cães de áreas rurais do Estado do Rio de Janeiro infestados por carrapatos Amblyomma cajennense, Amblyomma aureolatum, Amblyomma ovale e Rhipicephalus sanguineus.

\begin{tabular}{|c|c|c|c|c|c|}
\hline \multirow[b]{2}{*}{ Município } & \multicolumn{5}{|c|}{ Carrapatos } \\
\hline & $\begin{array}{c}\text { Amblyomma } \\
\text { cajennense }\end{array}$ & $\begin{array}{c}\text { Amblyomma } \\
\text { aureolatum }\end{array}$ & Amblyomma ovale & $\begin{array}{c}\text { Rhipicephalus } \\
\text { sanguineus }\end{array}$ & $\begin{array}{c}\text { Total de cães } \\
\text { examinados }\end{array}$ \\
\hline Seropédica & 0 & 0 & 0 & $1(6,67 \%)$ & 15 \\
\hline Itaguaí & $28(39,4 \%)$ & 0 & $3(4,2 \%)$ & $13(18,3 \%)$ & 71 \\
\hline Paracambi & $13(46,4 \%)$ & 0 & 0 & 0 & 28 \\
\hline Barra do Piraí & $1(4,0 \%)$ & $5(20,0 \%)$ & 0 & $2(8,0 \%)$ & 25 \\
\hline Piraí & $4(14,3 \%)$ & 0 & 0 & $2(7,1 \%)$ & 28 \\
\hline Miguel Pereira & $2(20,0 \%)$ & 0 & 0 & $4(40,0 \%)$ & 10 \\
\hline Mangaratiba & 0 & 0 & 0 & $4(18,2 \%)$ & 22 \\
\hline Total & $48(24,1 \%)$ & $5(2,5 \%)$ & $3(1,5 \%)$ & $26(13,6 \%)$ & 199 \\
\hline
\end{tabular}

Ciência Rural, v. 34, n. 1, jan-fev, 2004. 
Tabela 3 - Relação entre a presença de Amblyomma cajennense e Rhipicephalus sanguineus e a ocorrência de anticorpos anti-Borrelia burgdorferi latu sensu em cães de áreas rurais do Estado do Rio de Janeiro.

\begin{tabular}{|c|c|c|c|c|c|}
\hline \multirow{2}{*}{$\begin{array}{l}\text { Anticorpos contra- } \\
\text { Borrelia burgdorferi }\end{array}$} & \multicolumn{2}{|c|}{ Amblyomma cajennense } & \multicolumn{2}{|c|}{ Rhipicephalus sanguineus } & \multirow{2}{*}{$\frac{\text { Cães }}{\text { Total }(\mathrm{N})}$} \\
\hline & Ausência N (\%) & Presença N (\%) & Ausência N (\%) & Presença N (\%) & \\
\hline Negativo & $132(78,6)$ & $36(21,4)$ & $149(88,7)$ & $19(11,3)$ & 168 \\
\hline Positivo & $19(61,3)$ & $12(38,7)$ & $24(77,4)$ & $7(22,6)$ & 31 \\
\hline Total & $151(75,9)$ & $48(24,1)$ & $173(86,9)$ & $26(13,1)$ & 199 \\
\hline
\end{tabular}

No Brasil, a prevalência de $\boldsymbol{B}$. burgdorferi em cães foi pesquisada por SOARES et al. (1999a) que observaram prevalência de $20 \%$ e por JOPPERT et al. (2001) que encontraram 9,7\%. Assim, os resultados observados no presente estudo $(15,58 \%)$ estão próximos do que vem sendo reportado no Brasil e de acordo com a prevalência em áreas endêmicas de outros países. A prevalência uniforme de anticorpos anti-B. burgdorferi entre os diferentes municípios é um indicativo da presença de B. burgdorferi latu sensu em áreas rurais do Estado do Rio de Janeiro e de sua ampla distribuição.

Não houve diferença significativa entre a distribuição da ocorrência de anticorpos anti-B. burgdorferi $\mathrm{e}$ as faixas etárias a partir de seis meses de idade. Estes resultados estão de acordo com os observados em outros estudos. GREENE et al. (1988) encontraram cães positivos, pelo método de Imunofluorescência Indireta, em todas as faixas etárias, com maior prevalência em animais de um a cinco anos. MAGNARELLI et al. (1985) observaram maior número de casos positivos, na IFI, em cães com idade superior a quatro anos. JOPPERT et al. (2001) não verificaram diferença entre as faixas etárias de cães no Estado de São Paulo. MERINO et al. (2000) observaram animais soropositivos a partir de um ano de idade. Cães com idade inferior a seis meses podem não ter sido expostos ao agente, podem ter anticorpos colostrais em níveis muito baixo, ou não terem produzido anticorpos suficientes para serem detectados no teste ELISA. A partir desta idade, estímulos constantes podem manter a produção de anticorpos em níveis suficientes para serem detectados nos testes sorológicos (HOVIUS et al., 1999a).

No Brasil, cães de áreas rurais são parasitados principalmente por carrapatos do gênero Amblyomma (MASSARD et al., 1981; O' DWYER et al., 2001) que podem desempenhar papel importante na transmissão do agente para cães e também para seres humanos, enquanto em cães de áreas urbanas, R. sanguineus é o carrapato mais prevalente (MASSARD et al., 1981; RIBEIRO et al., 1997).
A presença de anticorpos contra $\boldsymbol{B}$. burgdorferi em cães de área rural do Rio de Janeiro é um indicativo da ampla distribuição do agente nessa área e do risco de ocorrerem casos humanos da doença na região. Por tratar-se de doença pouco conhecida no nosso meio, muitos casos podem não ser corretamente diagnosticados e tratados. Apesar de não ter havido associação entre a presença de carrapatos e a soropositividade para B. burgdorferi, deve-se considerar que a presença de anticorpos indica exposição prévia ao agente e que o animal infectado pode não estar mais sendo parasitado pelo carrapato responsável pela transmissão. Devido à ampla distribuição e baixa especificidade de carrapatos do gênero Amblyomma, principalmente nas áreas rurais, não se pode descartar a possibilidade de esses carrapatos serem responsáveis pela transmissão da borreliose de Lyme no Brasil. Assim, estudos devem ser conduzidos a fim de se conhecer a prevalência desse agente em outras localidades, sua patogenia e os vetores envolvidos em sua transmissão no Brasil.

\section{REFERÊNCIAS BIBLIOGRÁFICAS}

ARAGÃO, H.; FONSECA, F. Notas de Ixodologia. VIII. Lista e chave para os representantes da fauna ixodológica brasileira. Memórias do Instituto Oswaldo Cruz, v.59, p.115-130, 1961.

AZUMA, Y. et al. Canine Lyme disease: clinical and serological evaluations in 21 dogs in Japan. Veterinary Record, v.134, p.369-372, 1994.

BARBOUR, A.G. et al. Identification of an uncultivable Borrelia species in the hard tick Amblyomma americanum: possible agent of a Lyme disease-like illness. Journal of Infectious Disease, v.173, p.403-409, 1996.

BAUERFEIND, R. et al. Detection of Borrelia burgdorferi in urine form dogs by a nested polymerase chain reaction. Zentralbl Bakteriologie, v.287, p.347-61, 1998 .

FISHER, L.D.; BELLE, G.V. Biostatistic. A methodology for the health sciencies. New York : Wiley-Interscience, 1993. 991p. 
FONSECA, A.H. et al. Lyme borreliosis serology in cattle in Brazil. Revista Universidade Rural, Série Ciências da Vida, v.18, p.85-89, 1996

GOODMAN, L.A. Simultaneus confidence intervals for contrast among multinomial populations. Annals of Mathematic and Statistic, v.35, p.716-725, 1964.

GOODMAN, L.A. Simultaneus confidence intervals for multinomial proportions. Technometrics, v. 7, p.247-254, 1965.

GREENE, R.T. et al. Antibodies to Borrelia burgdorferi in dogs in North Carolina. American Journal of Veterinary Research, v.49, p.473-476, 1988 .

HOSKINS, J.D. Tick-borne zoonoses: Lyme disease, ehrlichiosis, and Rock Mountain spotted fever. Small Animal, v. 6, p.236-243, 1991.

HOVIUS, K.E. et al. A serological study of cohorts of young dogs naturally exposed to Ixodes ricinus ticks, indicates seasonal reinfections to Borrelia burgdorferi sensu latu. Veterinary Quarterly, v.21, p.16-20, 1999a.

HOVIUS, K.E. et al. Presence and distribution of Borrelia burgdorferi sensu latu species in internal organs and skin of naturally infected symptomatic and assymtomatic dogs, as detected by polymerase chain reaction. Veterinary Quarterly, v.20, p.54-58, 1999b

JOPPERT, A.M.; HAGIWARA, M.K.; YOSHINARI, N.H. Borrelia burgdorferi antibodies in dogs from Cotia county, São Paulo State, Brazil. Revista do Instituto de Medicina Tropical de São Paulo, v.43, p.251-255, 2001.

LEVY, S.A.; DREESEN, D.W. Lyme borreliosis in dogs. Canine Practice, v.17, p.5-14, 1992.

LINDENMAYER, J.M.; MARSHALL, D.; ONDERDONK, A.B. Dogs as sentinels for Lyme disease in Massachusetts. American Publication of Health, v.81, p.1448-1455, 1991.

MAGNARELLI, L.A. et al. Borreliosis in dogs from Southern Connecticut. Journal of American Veterinary Medicine Association, v.189, p.955-959, 1985.

MASSARD, C.A.; MASSARD, C.L.; RESENDE, H.E.B. Carrapatos de cães de áreas urbanas e rurais de alguns estados brasileiros. In: CONGRESSO BRASILEIRO DE PARASITOLOGIA, 6., 1981, Belo Horizonte, MG. Anais... Belo Horizonte : Sociedade Brasileira de Parasitologia, 1981. p.201.

MCKENNA, P. et al. Canine Lyme disease in Belgium. Veterinary Record, v.136, p.224-247, 1995.

MERINO, F.J. et al. Epidemiological characteristics of dogs with Lyme borrelioses in the province of Soria. European Journal of Epidemiology, v.16, p.97-100, 2000.
O'DWYER, L.H.; MASSARD, C.L.; SOUZA, J.C.P. Hepatozoon canis infection associated with dog ticks of rural areas of Rio de Janeiro state, Brazil. Veterinary Parasitology, v.94, p.143-150, 2001.

OLSON, P.E. et al. Canine as sentinels for Lyme disease in San Diego County, California. Journal of Veterinary Diagnostic Investigation, v.12, p.126-129, 2000.

RIBEIRO, V.L.S. et al. Espécies e prevalência das infestações por carrapatos em cães de rua na cidade de Porto Alegre, RS, Brasil. Ciência Rural, v.27, p.285-289, 1997.

SALINAS-MELENDEZ, J.A. et al. Serological survey of canine borreliosis. Revista Latinoamericana de Microbiologia, v.41, p.1-3, 1999.

SOARES, C.O. et al. Sorologia para borreliose em cães procedentes da Baixada Fluminense, Estado do Rio de Janeiro. Revista Brasileira de Medicina Veterinária, v. 21, p.111114, 1999a.

SOARES, C.O. et al. Ensaio imunoenzimático indireto na detecção de anticorpos homólogos da classe IgG contra Borrelia burgdorferi latu sensu em cães. Revista Brasileira de Medicina Veterinária, v.21, p.153-158, 1999b.

SOARES, C.O. et al. Borrelioses, agentes e vetores. Pesquisa Veterinária Brasileira, v.20, n.1, p.1-19, 2000.

STEERE, A.C. Lyme disease. New England Journal of Medicine, v.31, p.586-597, 1989.

STEFANCIKOV, A. et al. ELISA comparison of three wholecell antigens of Borrelia burgdorferi sensu latu in serological study of dogs from area of Koice, Eastern Slovakia. Annals of Agriculture, Environmental and Medicine, v.5, p.2530, 1998.

STRAUBINGER, R.K. et al. Clinical manifestations, pathogenesis and effect of antibiotic treatment of Lyme borreliosis in dogs. Wien Klin Wochenschr, v.110, p. 87481, 1998.

STRAUBINGER, R.K. PCR-Based quantification of Borrelia burgdorferi organisms in canine tissues over a 500-Day postinfection period. Journal of Clinical Microbiology, v.38, p.2191-2199, 2000.

WRIGHT, J.C. et al. Seroprevalence of Borrelia burgdorferi in dogs in Alabama, USA. Preventive Veterinary Medicine, v.31, p.127-131, 1997

YOSHINARI, N.H. et al. Perfil da borreliose de Lyme no Brasil. Revista do Hospital das Clínicas da Faculdade de Medicina, São Paulo, v.52, n.2, p.111-117, 1997. 